\title{
A Novel Grid-based Resource Management Framework for Collaborative e-Learning Environments
}

\author{
Makhan Singh \\ University Institute of Engineering and Technology \\ Panjab University, \\ Chandigarh - 160014, India
}

\author{
Sarbjeet Singh \\ University Institute of Engineering and Technology \\ Panjab University, \\ Chandigarh - 160014, India
}

\begin{abstract}
With the advancements in the fields of computing, communication and control, the collaborative e-Learning services are rapidly gaining popularity all over the world. Today, we have tools and technologies that enable rapid design and implementation of many of the abstract ideas/concepts needed for the successful realization of such services. The collaborative eLearning services can be categorized as generalized services or need specific services. While it is easy to construct need specific services with the help of sophisticated tools available today, it is increasingly complex to build generalized collaborative e-learning services. Among many of the challenging issues present in the design and implementation of generalized services, the major ones are e-Resources discovery, scheduling, security and overall management and administration. This paper presents a grid based resource management model for generalized collaborative e-Learning environments. The model proposes ways to discover, store, extract, protect and manage e-Resources in an effective and interoperable way. The implementation is going on in open source technologies using Linux, Apache, MySQL and PHP.
\end{abstract}

\section{Keywords}

Grid-based Resource Management, e-Learning Services, Collaborative e-Learning Environments, e-Resource discovery, security and management.

\section{INTRODUCTION}

Distributed systems can be defined as collection of heterogeneous, autonomous administrative domains that collectively work on a large single problem. Today distributed systems have many variations and a lot of computing technologies are prevalent today with major ones like peer-to-peer computing, autonomic computing, internet computing, pervasive computing, cloud computing and grid computing etc. The main objective of distributed systems is to share resources but these technologies have also enabled the creation of large collaborative environments which can be used for various purposes. Today we have the concepts and transitional implementations of virtual classrooms, virtual laboratories, virtual organizations, virtual research groups and virtual social networks etc. As a result of these advancements, the e-Learning environments have become a reality and are rapidly gaining popularity. Though it is possible to implement eLearning environments with the help of existing tools and technologies today, still to make the implementation more effective and optimized, a lot more work and research is needed to be done. The umbrella e-Learning implementation problem is of management of e-Resources which includes various sub-problems like e-Resource discovery, scheduling and security. One possible way to handle this problem in an efficient, effective and scalable way is by making use of grid technologies to manage e-Resources. Grid technologies enable us to build large virtual organizations that allow sharing of a variety of resources through the concept of grid services. Thus grid technologies can be explored to implement e-Learning environments because implementation of eLearning environments also poses the same problems that grid technologies are trying to handle. As e-Learning environment requires management of large pool of e-Resources in various forms which are scattered over different geographical locations, the usage of grid technologies become more sensible. A lot of work has already been done in grid technologies to handle similar issues so the same can be applied to implementation of collaborative e-Learning environments also. The collaborative eLearning services can be categorized as generalized and need specific services. The need specific e-Learning services are comparatively easier to implement as the requirements are well understood and it needs fewer and known issues to be tackled. On the other hand, the generalized e-Learning services are difficult to implement, require more issues to be addressed and furthermore there are many unknown issues also that come into picture with time. This paper presents a grid based resource management model for generalized collaborative e-Learning environments. The paper is organized as follows:

First section describes the distributed systems and their types. The collaborative e-Learning services and their types are also discussed. Second section throws light on background and related word in the area of collaborative learning. The systems like Moodle, NPTEL, ATutor, Blackboard, Claroline, MIT opencourseware etc. are discussed. Third section presents the proposed grid based resource management model for collaborative learning. Section four talks about implementation blueprint. Finally, section five concludes the paper with summary and future plans.

\section{BACKGROUND AND RELATED WORK}

This section explains the concepts and basic features of some of the most popular systems like Moodle, NPTEL, MIT OpenCourseWare, ATutor, Blackboard, Claroline, \& Gridcole etc.

Learning management system (LMS) [7] is a software application. Some LMSs are Web-based to facilitate access to learning contents and administration and on other hand some are performance management system which facilitates in employee appraisals, competency management, skill-gap analysis and 360 
degree reviews. LMS helps in educational, administrative and deployment requirements [7].

Moodle is Modular Object-Oriented Dynamic Learning Environment (Moodle) [8], [9] and is also known as virtual Learning Environment. It is a free and open source e-learning software platform. This platform is designed to create online courses. These courses are developed with a focus on interaction and collaborative construction of contents. This platform can be used in many type of environments for example in education, training and development or in business [8], [9].

NPTEL is National Programme on Technology Enhanced Learning (NPTEL) [10] and is a project which is introducing multimedia and web Technology to enhance learning of basic science and engineering concepts. The target groups for this project are students and faculty of engineering institution in India. Its educational goals are to make video lectures in a format appropriate for broadcasting through technology channel named Eklavya and make e-learning material available in web for video lectures in order to help in class room teaching. NPTEL developed curriculum based video courses for 100-110 existing and around 112 new courses which are encapsulated in digital video formats. It has also developed 116 web-based e-courses [10].

MIT OpenCourseWare (MIT OCW) is a Content Management System which is based on Microsoft Content Management Server 2002. This is considered as a large scale-scale web-based publication of Massachusetts Institute of Technology course materials. It is a web-based publication of virtually all MIT course content. OCW is open and available to the world and is a permanent MIT activity. There is no registration or enrollment process because $\mathrm{OCW}$ is not a credit-bearing or degree-granting initiative [11].

ATutor [12], [13] is an open source web-based learning content management system used to develop and deliver online courses. This is social networking environment which is designed using accessibility and adaptability in mind. Open source technology makes it effective tool for presenting instructional materials on web or independent online courses [12], [13].

Blackboard [14] learning system is a web-based server software platform. It can be installed on local servers or it can be hosted by Blackboard ASP solutions. It is mainly used to add online elements to courses which are traditionally delivered in face to face or in class room. It can also be used to develop completely online courses with little or no face to face meetings [14].

Claroline [15] is an open source e-Learning and e-Working platform. This allows instructors to build online courses and to manage learning and collaborative activities on the web. This platform is organized around the concept of spaces related to course. Different spaces provide a list of tools which helps to create learning contents, manage training activities and interact with learners [15].

Online Learning and Training (OLAT) [16], [17] is a web based application which supports any kind of online learning, teaching.it has been created especially for public institutions such as universities, academies or colleges. It is open source developed in java programming language [16], [17].
Sakai [18], [19] is open source software which is distributed under the Educational Licence. It is a Java based service-oriented application. Sakai is a community of academic institution, commercial organization and individuals, all work together to develop a common collaboration and Learning Environment [18], [19].

Gridcole [1] is a collaborative learning system which can be easily customized by instructors for their computer supported collaborative learning (CSCL). This is done by using OGSA grid services which may be provided by third parties. It is a grid computing environment that allows user with no technical skills easy access to grid resources and applications in order to provide effective support for collaborative learning. It also allows learners easy participation in collaborative learning designs using these applications. [1].

GIMOLOUS [2] is an e-learning system which is based on Open Grid Services Architecture (OGSA). This system is for education in environmental science domain. Moreover it does not provide support for collaborative activities and activity management [2].

ISIlab [3] is an Internet Shared Instrumentation Laboratory. it is a remote laboratory which is based on a grid infrastructure. This is used for teaching electronics. Students can also practice with electronics instruments and measurement methods to execute real experiments on analog and digital circuits. This also does not support collaborative learning and activity management [3].

DARE [4] is a collaborative learning environment. It introduces an activity theory based conceptual model to enable Computer Supported Collaborative Learning (CSCL) tool integration. [4].

COLEG [5] is a Collaborative Learning Environment within Grid. Its aim is to deliver the capacities offered by the Grid to the various actors, all the power of learning, collaboration and communication in an adaptable, heterogeneous and dynamic sight. With this, the user may at anytime and from anywhere be able to integrate tools and services as per his or her requirements of learning without the intervention by administrator or any technical persons [5].

\section{GRID BASED RESOURCE MANAGEMENT FOR COLLABORATIVE E-LEARNING}

Resource Management deals with number of challenging issues with important ones being resource discovery, resource scheduling, resource allocation, resource protection, resource monitoring and control. Today grids are mature enough to handle these issues effectively. The most notable resource management middleware solution is the Grid Resource Allocation Manager (GRAM). This resource management middleware provides robust job management services for users some of which includes job allocation, status management, data distribution and jobs start/stop configurations [6]. It provides a set of standard interfaces and components to collectively manage a job task and to provide resource information. This information can be used for various purposes. Following figure describes the basic architecture of GRAM. 


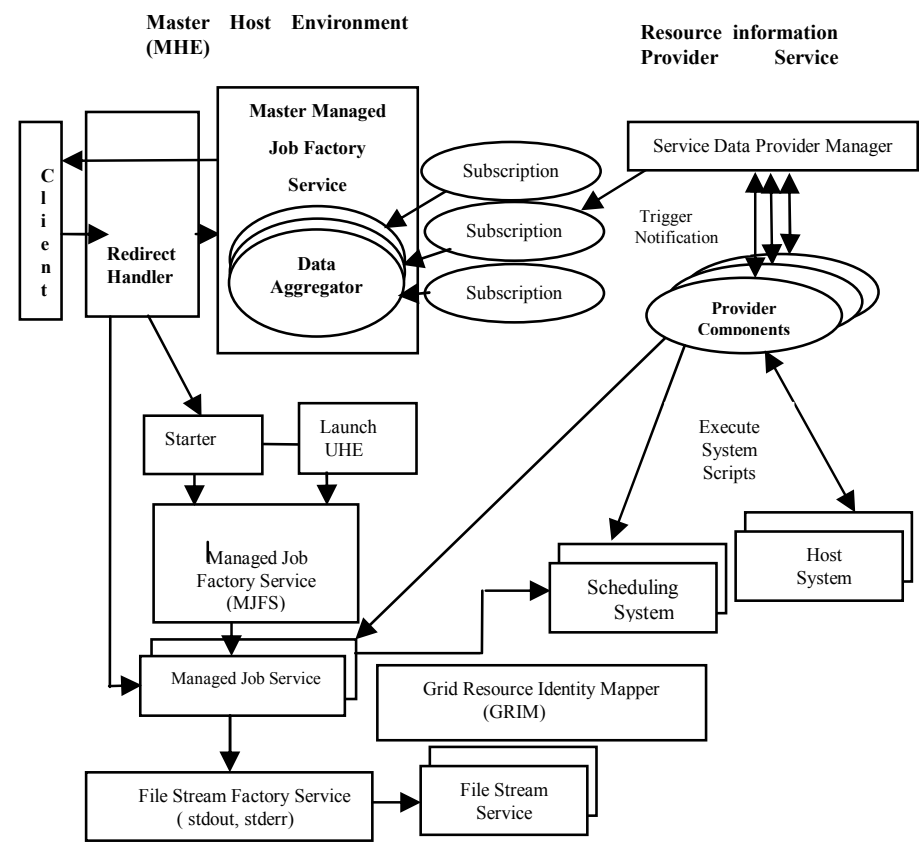

Fig1. GRAM Architecture [6]

Master Host Environment and User Host Environment enable separation of functionalities and give an improved abstraction on functions executed by each environment. The master host is direct point of contact for client. It provides information on aggregated resource state or status, and manages its user hosts start and launch services. The user host environment (UHE) executes all the jobs and provides specific abstraction capabilities and securities to execute job [6].

Master Managed Job Factory Service is responsible for receiving the client request on aggregated resource queries and subscriptions. It manages the aggregated service data by aggregation providers for resource status and through notifications received from resources [6].

Virtual Host Redirect Handler is the core component for redirecting all the calls to UHE. These Calls include creation of job and invoking of job operation [6].

When client request to execute a job, the virtual hosting engine directs the calls to starter. This java class is responsible for Security mapping, user validation and for ensuring that job is executing so that virtual host can redirect the calls to executing job service. When user host is not up and running, it uses the help of Lunch UHE java class to start that host under user's credentials [6].

Managed job Factory Service exposes CreateService method, which accepts an RSL-specified job. It then creates managed job instance for user. Moreover it acts as a local schedular, monitoring its status and sending notifications.

Managed Job Service will start two file streams factory services; one for job's stdout, and the other for the job's stderr.
File stream Factory Service/File stream service are helpful services to manage the data needed for the job execution. The factory service creates two file stream services: stdout and stderr. Each of these services has two service data results: the URL for the stream destination, and a flag to indicate the activity [6].

Grid Resource Identity Mapper (GRIM) service is executing in the UHE to create a user host certificate. The user host certificate is utilized for mutual authentication between MJS service and the client [6].

Resource Information Provider Service (RIPS) is a specialized notification service providing data about a scheduling system, file system, host [6].

\section{PROPOSED FRAMEWORK}

The framework described in Figure 1 has been used as the basis for the proposed collaborative e-Learning system. Following figure describes the major components of the proposed Gridbased Resource Management Model for collaborative e-Learning:

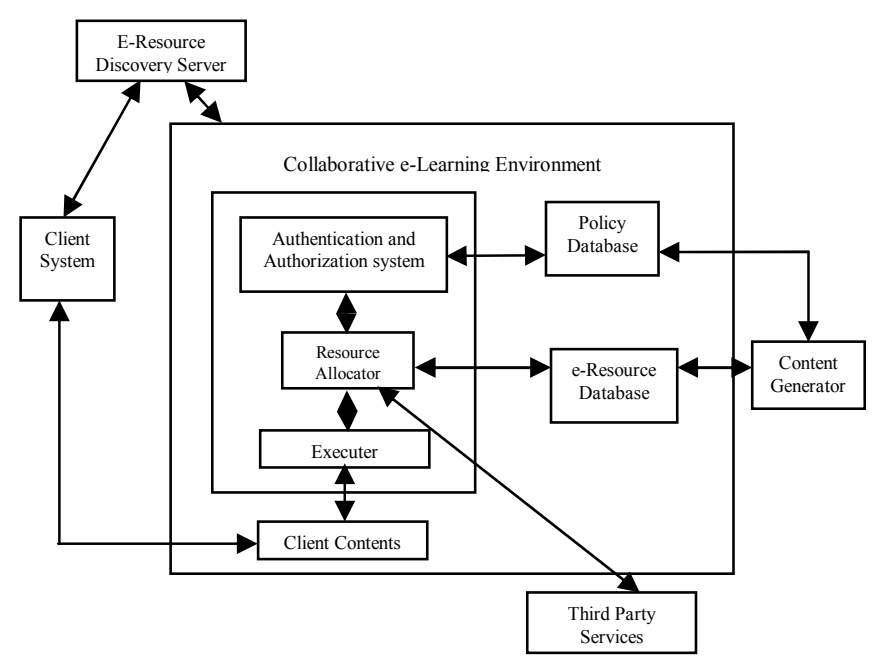

Fig 2. Proposed Grid-Based Resource Management Model

In this figure, the client system is comprised of browsers, application software or interactive terminals of any kind capable of sending data over the internet. The client request is first intercepted by authentication and authorization system at the Server end which checks client's authentication and authorization information with the policy database already maintained. Once the client is determined to be authentic and authorized to process his request, the Resource allocator is called and is supplied with the details of client request. Resource allocator examines the client request and depending upon the need, priority and other constraints, allocates the requisites number of resources for client usage. The resource information and resources are retrieved by Resource Allocator from e-Resource Database residing at the server end. The resources in the e-Resource database are generated by content Generator component of the system. Resource Allocator may also take the help of third party services to allocate exclusive resources or to allocate resources residing in 
other domains. Executor is a component that takes care of the execution part of the request. The client job is executed under the control of Executor. The client's contents are also generated under the control of Executor which are then flushed to client system. Client interaction with the server comes into picture after the discovery of server by the client system. The model shown in above figure requires the registration of the server with the eResource discovery service first, so that clients can find the resources available with that server and then the discovery of these resources by the client system. The Executor, Resource Allocator and Authentication and Authorization components have been clubbed as core functionalities. Other components of the system include Policy database and e-Resource database. While policy database is specifically concerned with authentication and authorization information of requester, e-Resource database contains exact information about various resources. e-Resource database may also be extended by third parties. A verifier component may also be placed between e-Resource database and third party services to check the contents generated by third parties. A separate content generator component is also shown in the figure which has the right to add contents in the e-Resource database and to associate the authentication and authorization policies of contents access with various requesters.

\section{IMPLEMENTATION DETAILS}

The implementation environment has been chosen to be consisting of Linux, Apache, MySQL and PHP as it is open source, mature, compatible and well tested. The various services of the model are being realized as grid services using GT4 (Globus ${ }^{\circledR}$ Toolkit 4). The interaction takes place through SOAP over HTTP. For Authentication and Authorization system implementation, the WS-Security and WS-* specifications are being used. For Resource Allocator and Executor, GT4 functionality has been used. The policy database has been created using XACML. The various services have been implemented as grid services.

\section{SUMMARY AND FUTURE SCOPE}

The paper presents a grid-based resource management model for collaborative learning environments. The proposed model is interoperable and capable of handling resource management issues related to discovery, scheduling, protection and administration. Currently, we are going though the implementation phase and in future we are planning to implement it with the trust and privacy features also. For this, the work in the areas for privacy and trust is also going on.

\section{REFERENCES}

[1] Bote-Lorenzo, M. L., Vaquero-Gonzalez, L. M. et. al., "GRIDCOLE: a Grid Collaborative Learning Environment", Proceedings of International Symposium on Cluster Computing and the Grid, 2004, pp. 105-112.

[2] Wesner, S., Wulf, K., and Müller, M., "How grid could improve E-learning in the environmental science domain", Proceedings of the 1st LEGE-WG international workshop on e-Learning and Grid technologies, Laussane, Switzerland, September 2002.

[3] Bagnasco, A. and Scappola, A. M., "A grid of remote laboratory for teaching electronics", Proceedings of the $2^{\text {nd }}$ LEGE-WG international workshop on e-Learning and Grid technologies, Paris, France, March 2003.

[4] Bourguin, G. and Derycke, A., "Integrating the CSCL activities into virtual campuses: foundations of a new infrastructure for distributed collective activities", Proceedings of the European Conference on Computer Supported Collaborative Learning, Euro-CSCL 2001, Maastricht, The Netherlands, Mar. 2001, pp. 123-130.

[5] Lafifi Y., Halimi K. and Hadjeris M., "COLEG: Collaborative Learning Environment within Grid", Journal of Computing and Information Technology, Vol. 18, No. 1, 2010 .

[6] Joshy Joseph and Craig Fellenstein, "Grid Computing", Pearson Education, 2004. 